\title{
Phase Formation and Microstructural Evaluation in V-Ti-Cr System Using Advanced Microscopy Analysis
}

\author{
Chanchal Ghosh ${ }^{1}$, Joysurya Basu ${ }^{2 *}$ and R Divakar ${ }^{1}$ \\ ${ }^{1 .}$ Metallurgy and Materials Group, Indira Gandhi Centre for Atomic Research, Kalpakkam, India. \\ 2. Department of Metallurgical Engineering, Indian Institute of Technology (BHU), Varanasi, India. \\ * Corresponding author: jbasu.met@iitbhu.ac.in
}

In the last couple of decades, there is a surge in the research on V-Ti-Cr alloys due to their advanced engineering applications at elevated temperatures [1-2]. The factors consisting of good fabricability, high temperature durability, heat load capacity, favorable safety and environmental characteristics, excellent compatibility with liquid metals and resistance to the irradiation damage qualify vanadium alloys as one of the candidate structural materials for fusion power applications as well as for hydrogen storage application [3-4].

However, the phase transformation behaviour of the constituent binaries and the ternary V-Ti-Cr system are not well understood in literature. Based on Miedema model, compositions along different phase field of $\mathrm{V}-\mathrm{Ti}-\mathrm{Cr}$ ternary systems have been identified and prepared by vacuum arc melting $-\mathrm{V}-4 \mathrm{Ti}-4 \mathrm{Cr}$ from the $\mathrm{V}$ rich corner of the ternary system to study stability of the solid solution phase, V-39Ti-54Cr from the Ti-Cr rich side of the diagram to study stability of amorphous / intermetallic phases, V-50Ti and V40Ti-20Cr from the V-Ti rich side of the alloy system to study presence of a miscibility gap [5]. These calculations have further been supported by ThermoCalc model and Pettifor structure map approach. Microstructural and microchemical evolution in this set of alloys has been studied using advanced transmission electron microscopy (TEM) techniques of phase contrast (with multislice simulation), STEM-HAADF, XEDS, EFTEM and EELS.

Detailed TEM studies confirm the stability of a $b c c$ solid solution phase at the V-rich corner of the V-Ti$\mathrm{Cr}$ ternary system. For a ternary extension of the binary $\mathrm{TiCr}_{2}$ Laves phase, formed by addition of $\mathrm{V}$ to the extent of 7 atom $\%$, zero loss phase contrast microscopy has been carried out to distinguish the atomic columns unambiguously. Results indicate that $\mathrm{Ti}$ and $\mathrm{Cr}$ atoms are not imaged simultaneously at all thickness - defocus combinations; this is further confirmed by phase contrast image simulations. The difference in the channelling behaviour of $\mathrm{Ti}$ and $\mathrm{Cr}$ atomic columns convoluted with the instrument $\mathrm{CTF}$ results in the disappearance of bright contrast for specific atom sites in the unit cell. Comparisons of the experimental and simulated electron intensity profiles confirm that $\mathrm{V}$ preferentially substitutes $\mathrm{Ti}$ rather than $\mathrm{Cr}$ from the regular lattice sites. This further confirms, $\mathrm{V}$-doped $\mathrm{TiCr}_{2}$ exists as a pseudobinary Laves phase with a modified stoichiometry of $(\mathrm{Ti}, \mathrm{V}) \mathrm{Cr}_{2}$ [6]. The calculated dopant formation energies using first principla calculations for both the $\mathrm{Ti}(0.29 \mathrm{eV})$ and the $\mathrm{Cr}(0.40 \mathrm{eV})$ site substitutions also suggest that $\mathrm{V}$ prefer to replace $\mathrm{Ti}$ rather than $\mathrm{Cr}$ from its regular lattice sites which is in confirmation with the microscopy observations.

For binary V-Ti alloys, the prediction of a phase separation tendency that reduces with addition of $\mathrm{Cr}$ by thermodynamic calculations is confirmed. Lamellar nanostructured domains with coherent interfaces seen in the phase contrast imaging of V-50Ti and V-40Ti-20Cr alloys, indicative of a phase separation event. STEM-HAADF studies indicate the compositional modulation normal to the lamellar domains and further examined through STEM-XEDS and STEM-EELS techniques. It is apparent from the results that the phase separation is essentially a binary event involving only $\mathrm{Ti}$ and $\mathrm{V}-\mathrm{Cr}$ is homogeneously 
distributed. The initial composition fluctuation wave in binary V-50Ti alloy is of wavelength $\sim 4-5 \mathrm{~nm}$. Gradually another wave with wavelength $\sim 18-20 \mathrm{~nm}$ initiates and finally this alone persists. Subsequent STEM and STEM based spectroscopy studies suggest that the composition modulation is governed by a spinodal decomposition mechanism and not by diffusion and growth kinetics [7].

As a result of the phase separation, a Ti rich domain evolves in the matrix, which becomes thermodynamically viable for the nucleation of $\omega$ phase. HRTEM images as recorded along $<1 \quad 1 \quad 0>$ and $<-113>$ zone axis of the $b c c$ phase confirms that in most of the cases the collapse is partial, yielding the trigonal $\omega$ phase. HRTEM analysis along with image simulation further shows the systematic absence of the bright atom contrast for the corner atoms of the $\omega$ phase. With the aid of quantitative electron microscopy an estimation of strain has been calculated along the interface of the $b c c$ and $\omega$ structured domains [8]. A combination of phase contrast and $\mathrm{Z}$ contrast microscopy coupled with electron spectroscopy imaging, phase contrast image simulation and atomic structure modelling have been successfully analyse the materials related issues of $\mathrm{V}-\mathrm{Ti}-\mathrm{Cr}$ alloys which is otherwise not possible through any of the conventional techniques [9].

References:

[1] RJ Kurtz et al., Journal of Nuclear Materials 329-333 (2004), p. 47.

[2] SJ Zinkle et al., Journal of Nuclear Materials 258-263 (1998), p. 205.

[3] M Tsukahara, Materials Transactions 52(1) (2011), p. 6.

[4] E Akiba and H Iba, Intermetallics 6 (1998), p. 461.

[5] C Ghosh et al., Materials Characterization 106 (2015), p. 292.

[6] C Ghosh et al., Philosophical Magazine 95(22) (2015), p.2403.

[7] C Ghosh et al., Acta Materialia 121 (2016), p. 310.

[8] C Ghosh et al., Journal of Materials Science 53 (2018), p. 13186.

[9] JB would like to acknowledge the financial support received from the Department of Science and Technology, Govt. of India by Award No EMR/2016/007512.
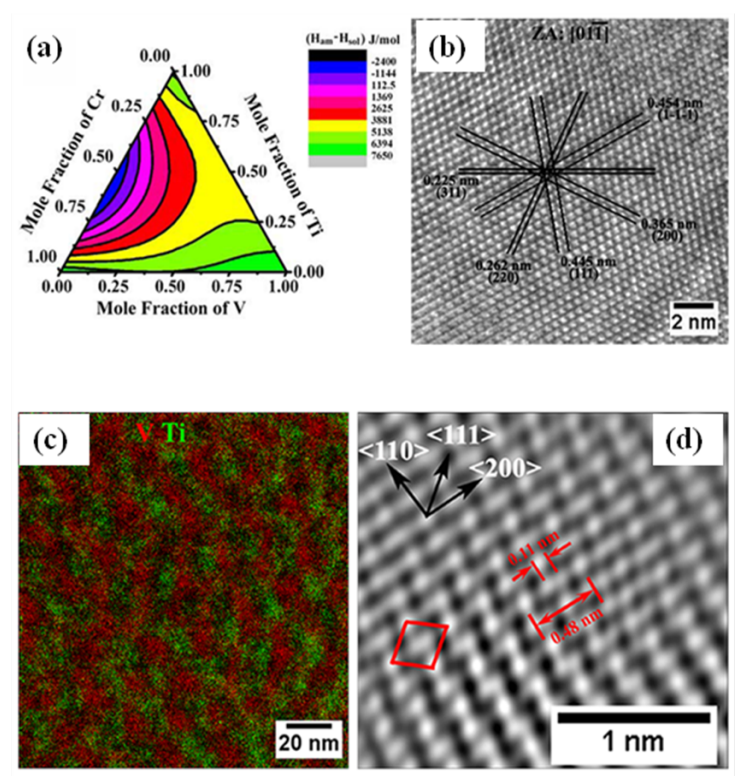

Figure 1. (a) Enthalpy contour plot as calculated from Miedema modal for the entire composition range of V-TiCr. (b) Zero loss phase contrast micrograph from V-substituted $\mathrm{TiCr}_{2}$ Laves phase. (c) EFTEM micrograph showing the composition modulation from a Spinodally decomposed region in a equiatomic V-Ti alloy. (d) Phase contrast micrograph showing the atomic arrangement in a partially collapsed $\omega$ domain. 